\title{
STATUS OF THE AIRIX ACCELERATOR
}

\author{
E. Merle, R. Boivinet, M. Mouillet, J.C. Picon, O. Pierret \\ CEA / DIF / PEM 51490 PONTFAVERGER-MORONVILLIERS - FRANCE \\ Ph. Anthouard, J. Bardy, C. Bonnafond, A. Devin, P. Eyl \\ CEA / CESTA BP n² 33114 LE BARP - France
}

\begin{abstract}
AIRIX is a linear induction accelerator that will be used for flash X-ray radiography. It will deliver a $20 \mathrm{MeV}$, $3.5 \mathrm{kA}, 60 \mathrm{~ns}$ electron beam. This accelerator is now under installation at PEM. The injector, that produces a $3.5 \mathrm{kA}, 4 \mathrm{MeV}, 60 \mathrm{~ns}$ electron beam and 16 induction cells powered by 8 high voltage generators $(250 \mathrm{kV}$ per cell) are already operational. At this point, the AIRIX LIA has the same dimension than the PIVAIR prototype installed at CESTA [1]. This paper relates the electron beam characterization that we have made to compare the two accelerators. Those experiments consist first, on beam imaging to validate beam transport code at three points on the beam line. In a second time we measure the energy of the beam with the time-resolved spectrometer to control the acceleration of the beam and to precisely tune the chronometry of the machine. Finally, we measure the emittance of the beam with the Pepper-pot method to compare the value before and after acceleration. We will present also the accelerator update and the experiments we plan to do when the 64 induction cells and the $32 \mathrm{H}$.V. generators will be installed.
\end{abstract}

\section{INTRODUCTION}

The installation of the AIRIX induction accelerator is going on at Moronvilliers. An experimental break has been realized during two months (december 98, january 99) and consisted to work with the injector and 16 induction cells. Different aims were expected:

- the validation of the technical choices for the machine

- the complete electron beam characterization after the nominal acceleration.

- the validation of the beam transport calculations

- the extrapollation to the AIRIX performances

This campaign is still under analysis, but we present here the first results. In a first part, we expose the beam initial characteristics measurements. The high voltage performances of the induction cells are related in the second point, and we expose also the associated energy spectrums. In the two following paragraphs, we present respectively, the beam emittance, and the beam radius measurement for different transport calculations.

\section{INJECTOR CHARACTERISTICS}

The complete electron beam characterization has been made for two running points of the injector: (4.0 MeV; $3.5 \mathrm{kA})$ and (3.6 MeV; $3.0 \mathrm{kA})$ [2]. The time-resolved energy spectrum [3] shows that, for a $\pm 1 \%$ energy spread, the flat top duration is $60 \mathrm{~ns}$ (fig. 1).

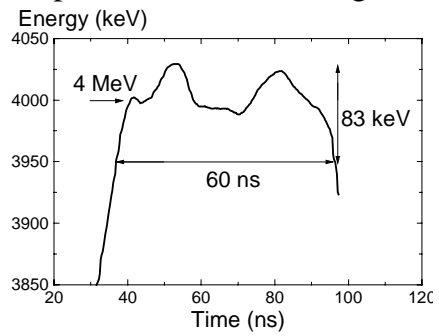

Figure 1: injector time-resolved energy spectrum

The emittance $(\varepsilon)$ measurement has been made with the Pepper-pot method [4].

\begin{tabular}{|c|c|c|}
\hline$\varepsilon(\pi \mathrm{mm} \mathrm{mrad})$ & $\mathrm{X}$ axis & Y axis \\
\hline $4.0 \mathrm{MeV}$ & 1150 & 1181 \\
\hline $3.6 \mathrm{MeV}$ & 977 & 1001 \\
\hline
\end{tabular}

Table 1 : Emittance values

measured with the pepper-pot method

We have choosen to use the normalized geometrical emittance. The 'global' emittance value, published until now, is higher because the emittance diagram is distorted by the pepper-pot focalization effect [4].

The determination of the beam initial parameters (radius $\mathrm{R}_{0}$ and envelope slope $\mathrm{R}_{0}{ }_{0}$ ) is made with the classical three gradients method. The electron beam visualization is made by an intensified and gated camera observing Cerenkov radiation obtained by the beam interaction with a $5 \mu \mathrm{m}$ thin aluminized mylar foil.

\begin{tabular}{|c|c|c|c|c|}
\hline Energy & \multicolumn{2}{|c|}{$3.6 \mathrm{MeV}$} & \multicolumn{2}{c|}{$4.0 \mathrm{MeV}$} \\
\hline Axis & $\mathrm{X}$ & $\mathrm{Y}$ & $\mathrm{X}$ & $\mathrm{Y}$ \\
\hline $\mathrm{R}_{0}(\mathrm{~mm})$ & 32.4 & 33.4 & 29.7 & 30.04 \\
\hline $\mathrm{R}_{0}{ }_{0}(\mathrm{mrad})$ & 75.7 & 77.5 & 70.21 & 70.03 \\
\hline$\varepsilon(\mathrm{p} \mathrm{mm} \mathrm{mrad})$ & 1013 & 826 & 1190 & 1025 \\
\hline f.o.m. $(\%)$ & 2.1 & 2.1 & 1.94 & 1.45 \\
\hline
\end{tabular}

Table 2 : Beam initial parameters

The factor of merit (f.o.m.) evaluates the good adequation between the calculated values of the beam diameter and the measured values. In a perfect case, (measured and calculated values are identical) the f.o.m. is zero. 
We can notice, that the emittance values obtained with the two different methods are very closed. More, in the two directions, $\mathrm{X}$ and $\mathrm{Y}$ axis, $\mathrm{R}_{0}$ and $\mathrm{R}_{0}$ are very closed too. The beam seems to be symetrical along the electron beam propagation axis $(\mathrm{z})$.

\section{INDUCTION CELLS}

The induction cells installed on the AIRIX machine are vacuum technology cells [5]. They are powered by high voltage generators ( $250 \mathrm{kV}$ per cell, 2 cells per generator). The ferrite section has been increased by $9 \%$, in comparison with the PIVAIR cells, in order to delay ferrite saturation and decrease overvoltage stresses.

A high accuracy energy measurement of the accelerated beam, has been made with the time-resolved energy spectrometer. On the figure 2 we can observe a typical H.V. signal measured on the cells, and on the figure 3 the associated spectrum.

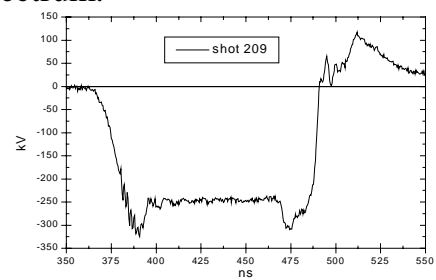

Figure 2: induction cell high voltage signal

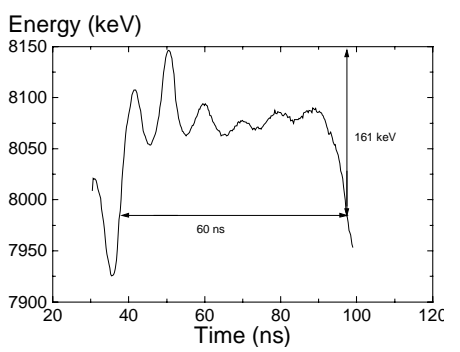

Figure 3: accelerated beam energy spectrum

The two peaks on the cells high voltage signal are caused by the presence of the beam that has a smaller duration, and that procures a different impedance of the cell. We can see also those picks because the ferrite material is not yet saturated.

The first peak that we can see on the spectrum (8020 keV) corresponds to the rise time of the beam pulse. At this time, the beam current is smaller than the $3.5 \mathrm{kA}$ nominal value and the H.V. seen by those electrons is higher than the $250 \mathrm{kV}$ nominal value (peaks on the high voltage signal).

The presence of the two peaks (figure 2), facilitates the synchronisation of the machine. More, it can absorb the 5 ns of the high voltage power units jitter.

The figure 3 shows that the energy nominal value is $8 \mathrm{MeV}$, and the $\pm 1 \%$ energy spread is preserved although the shape has been singularly modified, compared to the injector energy spectrum one (figure 1).

We can act on this shape by tuning the delay between the beam and the high voltage accelerating pulse. In the two cases presented here (figure 4 and 5) with the spectrum and the associated cells H.V. signals, the difference between the delays is $10 \mathrm{~ns}$. We observe that the first risetime peak can disappear completely (shot 282), but the two following ones have too high level. In opposite, the rise-time peak can be integrated to the spectrum and makes it wider.

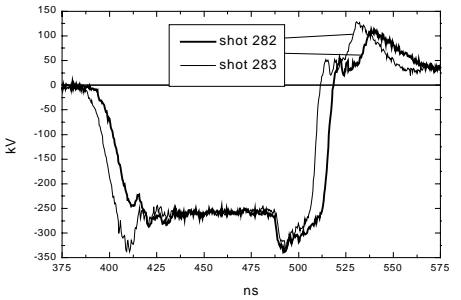

Figure 4: Cells H.V. signal for two shots with $10 \mathrm{~ns}$ different delays between the injector and accelerator

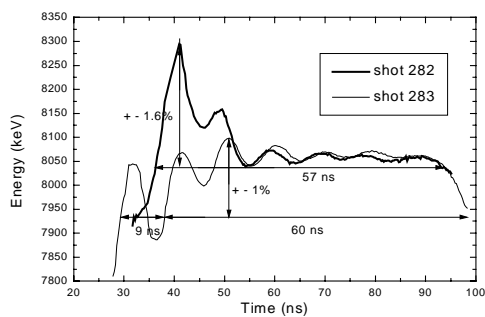

Figure 5: Spectrum associated to the shots 282 and 283

In routine, we will work with a symetrical H.V. signal, to preserve the minimum energy spread, during the $60 \mathrm{~ns}$ pulse width (figure 2,3).

\section{BEAM EMITTANCE}

The normalized beam emittance has been measured with the Pepper-pot method.

\begin{tabular}{|c|c|c|}
\hline$\varepsilon(\pi \mathrm{mm} \mathrm{mrad})$ & $\mathrm{X}$ axis & Y axis \\
\hline $8.0 \mathrm{MeV}$ & 1376 & 1273 \\
\hline $7.6 \mathrm{MeV}$ & 1330 & 1266 \\
\hline
\end{tabular}

Table 3 : emittance measurement after $4 \mathrm{MeV}$ acceleration The emittance increase is around $200 \pi \mathrm{mm} \mathrm{mrad}$, and is the same as the one measured on the PIVAIR machine.

\section{ELECTRON BEAM TRANSPORT}

To calculate the electron beam transport, we use the classical envelop equation:

$$
\begin{gathered}
\mathrm{R}^{\prime \prime}+\mathrm{k}^{2} \mathrm{R}-\frac{\mathrm{K}}{\mathrm{R}}-\frac{\varepsilon^{2}}{\beta^{2} \gamma^{2} \mathrm{R}^{3}}=0 \\
\mathrm{~K}=\frac{2 \mathrm{I}}{17.045 \beta^{3} \gamma^{3}} \text { (I in kAmps); } \mathrm{k}=\frac{\mathrm{e} \mathrm{c} \mathrm{B}_{\mathrm{z}}}{2 \beta \gamma \mathrm{mc}^{2}}
\end{gathered}
$$

The ENV code [6] takes into account the effect of the ferrite cores in the calculation of the axial magnetic field. We have improved several beam transport configurations, and each time, we have measured the beam diameter at the exit of the 16 th cell.

In a first case (figure 6), we have transported the $7.6 \mathrm{MeV}$ $(3.6 \mathrm{MeV}+16 * 250 \mathrm{keV})$ electron beam on a $40 \mathrm{~mm}$ diameter (400 Gauss axial magnetic field). In the second case (figure 7 ), the beam has a $20 \mathrm{~mm}$ diameter along the 
accelerator (700 Gauss axial magnetic field), and final energy is $8 \mathrm{MeV}$.

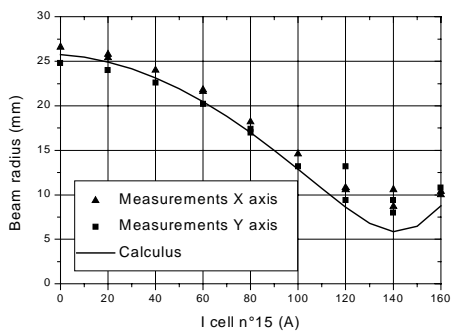

figure 6: variation of beam diameter with magnetic field on the $15^{\text {th }}$ cell

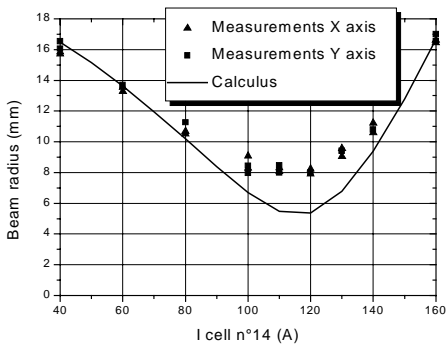

figure 7: variation of beam diameter with magnetic field on the $14^{\text {th }}$ cell

We can distinguish two parts on those curves. In a first part $(\mathrm{I}<100 \mathrm{~A})$, the electron beam is homogeneous, and has a classical square profile. The correlation between measurements and calculus is good. With those currents, the beam has not been focalised (small beam-waist) before the target. We will be generally in that case to transport the beam throughout the machine.

In the second part of the curves, the beam has been focalized before the target, and then the difference between the measured and calculated radius grows up to $3 \mathrm{~mm}$. Perhaps in that case the envelop equation used is not complete, or there are $2 \mathrm{D}$ effects. The beam profile is not square for those points. Different numerical and analytical investigations have been initialized to understand those differences.

Nevertheless, those results prove that the electron beam transport on the AIRIX machine can be calculated with the ENV code that is well predictive in most of cases.

We have improved also the centering procedure to have a centered beam all along the machine. One BPM (Beam Position Monitor) is installed on each four cells block. To optimize the entrance of the beam in the accelerator, we have installed another BPM, $50 \mathrm{~cm}$ before the one placed on the first cell. With those two measurements, we can obtain a centered beam with a minimum centroïd slope. If the centering procedure at the entrance of the accelerator is made rigorously, the beam is centered in a $2 \mathrm{~mm}$ diameter circle all along the machine.

On the figure 8, we have reported the first 7 BPM measurements. Each point per BPM corresponds to one shot, and represents the mean value of the beam centroïde motion calculated during $40 \mathrm{~ns}$ on the flat top of the current pulse. Day after day, the third and the last shot of the day are identical (same parameters for the machine). With this method, we can evaluate the machine stability, as shown on the next figure where 9 shots, corresponding to 4 days, are plotted.

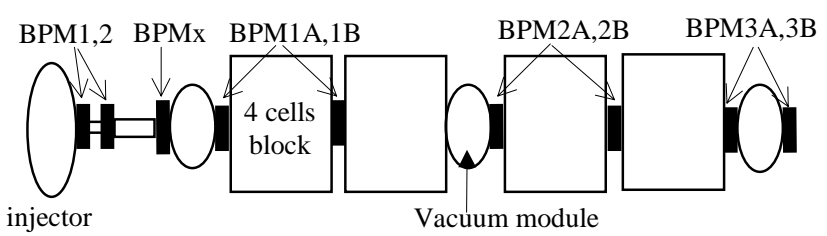

Schema of the 'AIRIX 16 cells' experiment

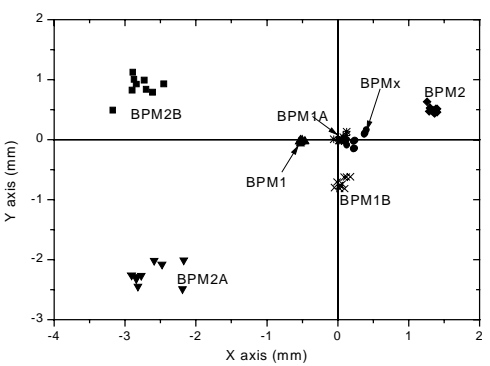

figure 8: centroïd beam measurement

The space occupied by the centroïd is larger at the end of the machine. This is due to the reliability of the power supplies that delivers current to the steering magnet. Modifications are under realization to correct this defect.

\section{CONCLUSION}

The "AIRIX 16 cells experiment", has been complete and comprises also BBU measurements. Several parametric variations in the magnetic field configuration have been tested. The results are now under analysis and will be published subsequently.

At this time, the $32 \mathrm{H} . \mathrm{V}$. units are already installed on the machine, and 36 induction cells too. The machine will be complete next summer. The acceptance test will consist essentially in the time-resolved energy measurement, and a reproducibility of the electron beam characteristics test. We plan to make the first hydrotest with radiographic diagnostic in december 99.

\section{REFERENCES}

$1 \mathrm{Ph}$. Anthouard et al., "AIRIX prototype technological results at CESTA”, Proc.. of PAC 97.

2 E. Merle et al., "Installation of the AIRIX induction accelerator", Proc. of LINAC 97.

3 D. Villate et al., "AIRIX alignment and high current electron beam diagnostics", Proc of PAC 95.

$4 \mathrm{C}$. Bonnafond et al. "Optical and time-resolved diagnostics for the AIRIX high current electron beam.”, Proc. of DIPAC 97

$5 \mathrm{Ph}$. Anthouard et al., "AIRIX and PIVAIR accelerator status"' Proc. of Beams 98.

6 J. Bardy - ENV "Envelop electron bem transport code", Private communication 